\title{
Necrotizing fasciitis after cesarean section
}

\author{
Alejandro González Castro · J. Carlos Rodriguez-Borregan · Tomas Obeso • \\ Alvaro Castellanos · Amelia Perez-Ceballos · José Ramón de Miguel Sesmero
}

Published online: 1 April 2008

(C) Springer-Verlag 2008

\section{Erratum to: Arch Gynecol Obstet DOI 10.1007/s00404-007-0509-z}

Unfortunately, the name of the corresponding author was not published correctly in the address.

A. González Castro ( $₫)$ · J. C. Rodriguez-Borregan ·

T. Obeso · A. Castellanos · A. Perez-Ceballos

Intensive Care Department,

Universitary Marques de Valdecilla Hospital,

Avda. Marques de Valdecilla s/n, 39011 Santander, Spain

e-mail: jandro120475@hotmail.com
J. R. de Miguel Sesmero

Gynecology and Obstetrics Department, Hospital Marqués de Valdecilla, Santander, Spain

The online version of the original article can be found under doi:10.1007/s00404-007-0509-z.

A. González Castro $(\bowtie) \cdot$ J. C. Rodriguez-Borregan .

T. Obeso - A. Castellanos - A. Perez-Ceballos

Intensive Care Department,

Universitary Marques de Valdecilla Hospital,

Avda. Marques de Valdecilla s/n,

39011 Santander, Spain

e-mail: jandro120475@hotmail.com

J. R. de Miguel Sesmero

Gynecology and Obstetrics Department,

Hospital Marqués de Valdecilla,

Santander, Spain 